\title{
Aprendizaje significativo relacionado con la formación académica en la Educación Superior
}

\author{
Meaningful learning related to academic training in Higher Education
}

Aprendizagem significativa relacionada á formação acadêmica no Ensino Superior

\section{Narcisa Cecilia Castro Chávez \\ narcisa.castroc@ug.edu.e}

Universidad de Guayaquil, Guayaquil, Ecuador

\section{Silvia Beatriz García Estupiñán}

sgarcia@uagraria.edu.ec

Universidad Agraria del Ecuador, Guayaquil, Ecuador
El aprendizaje significativo se muestra como una novedosa alternativa en la formación académica, ya que permite la creación de un conocimiento sólido y duradero sobre un determinado tema. El objetivo del estudio fue analizar el aprendizaje significativo desde una perspectiva de relectura en función de las transformaciones de la sociedad actual. La metodología fue tipo descriptiva desde un enfoque cualitativo. Los resultados conllevaron a propiciar un proceso personal de construcción del conocimiento. Se concluyó que en el proceso de orientación del aprendizaje, es de vital importancia conocer la estructura cognitiva del alumno de la Educación Superior. Emerge la importancia por saber cómo se adquieren los conocimientos para cuyo efecto tiene relevancia central el proceso de desarrollo cognoscitivo y la estructura cognoscitiva del sujeto.

Palabras clave:

Sociedad; construcción del conocimiento; estructura cognitiva; aprendizaje significativo; Educación superior
Meaningful learning is shown as a novel alternative in academic training, since it allows the creation of solid and lasting knowledge on a certain topic. The objective of the study was to analyze meaningful learning from a rereading perspective based on the transformations of today's society. The methodology was descriptive from a qualitative approach. The results led to promote a personal process of knowledge construction. It was concluded that in the learning orientation process, it is vitally important to know the cognitive structure of the Higher Education student. The importance of knowing how knowledge is acquired emerges, for whose effect the process of cognitive development and the cognitive structure of the subject have central relevance.

Key words: Society; knowledge construction; cognitive structure; significant learning; Higher education
A aprendizagem significativa mostra-se como uma alternativa inédita na formação acadêmica, pois permite a criação de conhecimentos sólidos e duradouros sobre um determinado tema. O objetivo do estudo foi analisar a aprendizagem significativa a partir de uma releitura a partir das transformações da sociedade atual. A metodologia foi descritiva de abordagem qualitativa. Os resultados propiciaram um processo pessoal de construção do conhecimento. Concluiu-se que no processo de orientação à aprendizagem é de vital importância conhecer a estrutura cognitiva do aluno do Ensino Superior. Surge a importância de saber como o conhecimento é adquirido, para cujo efeito o processo de desenvolvimento cognitivo e a estrutura cognitiva do sujeito têm relevância central.

Palavras-chave:

Sociedade; construção do conhecimento; estrutura cognitiva; aprendizagem significativa; Educação superior 


\section{INTRODUCCIÓN}

Aunque en la actualidad la enseñanza en la Educación Superior ha tomado un grado de calidad muy importante y relevante al momento de impartir una cátedra, se considera muy necesario aplicar estrategias que ayuden al estudiante a desarrollarse de la mejor manera, para lo cual una de las metodologías de poder enseñar podría ser al aprendizaje significativo.

Elaprendizajesignificativosemuestracomouna novedosa alternativa en la formación académica, ya que permite la creación de un conocimiento sólido y duradero sobre un determinado tema. Es preciso mencionar que las estrategias metodológicas permiten a los estudiantes indagar, aclarar dudas y cuestionar conceptos con el propósito de hacerse de un conocimiento integral. La siguiente investigación pretende explorar y comprender la importancia que tiene el aprendizaje significativo en la formación académica del profesional en la educación superior, ya que es un profesional requiere del aprendizaje de conocimientos de manera sólida.

Aprendizaje como define (Gagné, 1965) “un cambio en la disposición o capacidad de las personas que puede retenerse y no es atribuible simplemente al proceso de crecimiento" p.9; es decir, los procesos cognitivos involucrados se van precisando y conservando. Otra definición de "el aprendizaje se ocupa básicamente de tres dimensiones: como constructo teórico, como tarea del alumno y como tarea de los profesores; esto es, el conjunto de factores que pueden intervenir sobre el aprendizaje como cambios continuos de la persona en relación al algún objetivo" (Zavala, 1995, p.17).

Knowles (se basa en la definición de Gagné, Hartis y Schyahn, 2001) expresa que el aprendizaje es en esencia un cambio producido por la experiencia, pero distinguen entre el aprendizaje como producto, que pone en relieve el resultado final o el desenlace de la experiencia del aprendizaje; el aprendizaje como proceso, que destaca lo que sucede en el curso de la experiencia de aprendizaje para posteriormente obtener un producto de lo aprendido; y el aprendizaje como función, que realza ciertos aspectos críticos del aprendizaje, como la motivación, la retención, la transferencia que presumiblemente hacen posibles cambios de conducta en el aprendizaje humano.

Parra (2016) define el aprendizaje como el proceso a través del cual un estudiante adquiere conocimientos los va a ir perfeccionando a lo largo del tiempo por medio de nuevas experiencias, descubrimientos o avances.

Aprendizaje significativo es un tipo de aprendizaje en el cual un alumno "recibe el contenido de forma coherente, clara y organizada" (Escayola \& Vila, 2005, p. 72) lo que le permite obtener un conocimiento significativo y una actitud positiva al aprender. Según Canalejas, et-al (2005), el estilo de aprendizaje se define como la tendencia a través de la cual un individuo aprende, siendo estos el estilo activo, estilo reflexivo, estilo teórico, y estilo pragmático.

Enseñanza se refiere a la transmisión o el compartir de determinados conocimientos - previa planificación - por medio de métodos, técnicas y recursos que buscan el aprendizaje de por vida (Parra, 2016). Formación se define como "la capacidad de instruir, adoctrinar y amaestrar con reglas o preceptos" aun individuo (Parra, 2016, p. 45).

Según Kantor (1978), menciona que la educación es un proceso de socialización planificada lo cual facilita el desarrollo de la inteligencia y el aprendizaje; postula que la educación debe ser entendida no como contenidos formales sino como el establecimiento de actividades funcionales de una manera planificada, así, el aprendizaje se considera como la capacidad 
intelectual donde se desarrolla enseñando el cómo y no el qué al realizar ciertas actividades en situaciones específicas.

La Educación Superior tiene como objetivo la formación de capacidades y actitudes de los individuos para su integración a la sociedad como seres que sean capaces de regular el status quo y a la vez puedan transformar la realidad social en pos de los valores vigentes en un momento histórico determinado. Por tanto, la tarea de la educación superior es (Ibañez, 1994)“la formación de profesionales competentes; individuos que resuelvan creativamente, es decir, de manera novedosa, eficiente y eficaz, problemas sociales" (p. 104).

Así, las instituciones de educación superior, son las encargadas de la educación en los jóvenes y las características de éstas; están íntimamente relacionadas a la calidad de la formación de sus estudiantes, considerando que la calidad hace referencia a un sistema donde los principales factores son los individuos quienes son capaces de organizarse de forma eficiente para alcanzar las expectativas de la organización educativa (Guerrero, 2003), por ello, su función está dirigida al desarrollo de la creatividad e innovación en ellas mismas, propiciando un ambiente educativo que además de solucionar problemas sociales actuales junto con los alumnos, también ayuden a preparar mejores profesionistas para el futuro.

Según la UNESCO, única institución especializada de las Naciones Unidas que dispone de un mandato en educación superior y por esta razón, facilita la elaboración de políticas de base empírica en materia de enseñanza superior. En conformidad con la meta 43 del objetivo del desarrollo sostenible 4: “Para 2030, asegurar el acceso en condiciones de igualdad para todos los hombres y las mujeres a una formación técnica, profesional y superior de calidad, incluida la enseñanza universitaria", la UNESCO proporciona apoyo técnico a los Estados Miembros a fin de que puedan examinar sus estrategias y políticas relativas a la educación superior en aras de mejorar el acceso equitativo a una enseñanza superior de calidad y de reforzar la movilidad académica y la responsabilidad.

\section{MÉTODO}

La siguiente indagación está inscrita dentro del tipo de investigación descriptiva, ya que se pretende explicar de manera analítica lo relacionado al aprendizaje significativo y la formación académica desde un enfoque cualitativo no experimental ya que el objetivo principal de esta investigación es la observación y análisis del tema en cuestión planeado desde una perspectiva subjetiva (Baptista, Fernández, \& Hernández, 2014).

Según Baptista, Fernández y Hernández (2014), con la investigación descriptiva se "busca especificar propiedades y características importantes de cualquier fenómeno que se analice" (p. 92), al tiempo que expone que la investigación desde un enfoque cualitativo "busca principalmente la "dispersión o expansión" de los datos e información" (p. 10) para que un investigador "se forme creencias propias sobre el fenómeno estudiado", es decir ,se recurre a la investigación bibliográfica con el propósito de adquirir conocimientos subjetivos sobre un determinado tema.

Este tipo de aproximación "se basa en métodos de recolección de los datos no estandarizados" (Baptista \& et-al, 2014, p. 19), con el propósito de "obtener las perspectivas y puntos de vista de los participantes" (Baptista \& et-al, 2014, p. 19). Cabe señalar que el enfoque cualitativo admite un proceso de indagación interpretación flexible, ya que su propósito es "reconstruir la realidad tal como la observan los actores de un sistema social definido previamente" (Baptista \& et-al, 2014, p. 19). 


\section{RESULTADOS}

El aprendizaje significativo es un tipo de aprendizaje en el cual un individuo relaciona conocimientos y experiencias con nuevos saberes para generar ciertas habilidades por medio de la construcción de conocimientos propios desde una posición activa. Este tipo de aprendizaje se caracteriza por la relación que se suele realizar entre experiencias y nuevos conocimientos, para la construcción de nuevos contenidos que le permiten generar ciertas habilidades desde una posición activa (Parra, 2016). Este aprendizaje puede considerarse como una manera para el fácil aprendizaje debido a que se busca explotar el interés que tiene un alumno por aprender un determinado tema, lo que le permite interactuar con el docente de una manera activa, ya que busca convertirlo en participe de la actividad que se lleve en el aula, pues se busca que los alumnos proporcionen criterios, ideas y opiniones que coadyuven a la formación de un conocimiento propio que le permitirá un aprendizaje más sólido, objetivo que se busca hoy en día por la educación superior.

El aprendizaje significativo debe ser constructivo, activo, conceptualizado, social y reflexivo (Escayola \& Vila, 2005). En este sentido, si se pretende conseguir un aprendizaje sólido y duradero es primordial cumplir con cinco características básicas:

1. En primer lugar, se debe propiciar un proceso personal de construcción del conocimiento.

2. Se debe aplicar lo aprendido.

3. Se debe determinar el contexto espacio/ tiempo.

4. Se debe compartir el conocimiento de manera grupal con el profesor y los alumnos.

5. Se debe relacionar sobre lo aprendido.
Según Díaz Barriga y Hernández Rojas (2004), las fases de aprendizaje significativo son la fase inicial, intermedia, terminal la fase inicial se produce en el momento en que el alumno capta la información, sin embargo, esta presenta una estructura conceptual, dicho de otra manera, el alumno memoriza o interpreta la información y a continuación a construyendo de manera esquemática el conocimiento. En esta que el estudiante empieza a construir un conocimiento global que le permite aprender, en lo cual emplea el conocimiento esquemático adquirido, al tiempo que empieza a producir analogías con contenidos conocidos con anterioridad, creando un nuevo conocimiento basado en experiencias anteriores y relaciones actual.

La fase intermedia se realiza durante que el estudiante, comience a establecer puentes entre las ideas aisladas y va creando mapas cognitivos y esquemas sobre el conocimiento de manera progresiva, en otras palabras, el alumno ya empieza a llegar a conclusiones relativamente más claras, sin embargo, este conocimiento no es aún exacto, ya que, aunque la información generada le permite tener dominio del conocimiento, faltan reflexiones sobre el tema. En esta etapa el contenido de empieza a desligar de la información original, la creación de redes semánticas y mapas conceptuales.

En esta última etapa los conocimientos ya son comprendidos con mayor claridad, el conocimiento es más específico y propio en relación a la anterior. Es en esta fase donde se suele mostrar mayor interés por el tema y el aprendizaje producto de los cambios que se producen en la realización de las tareas ya que se realizan variantes, arreglos y ajustes. En este nivel se generan interrelaciones entre los conocimientos es decir el aprendizaje se vuele significativo. 
El aprendizaje significativo posee dimensiones que permite que el estudiante adquiera e incorpore conocimiento adquiridos a su estructura cognitiva siendo esas dimensiones la recepción y la repetición. Sin embargo, se estima que en el aprendizaje significativo el descubrimiento sólo se produce por repetición, sin embargo, se ha podido establecer que el aprendizaje significativo es también producto de la repetición del conocimiento. Cabe destacar que el aprendizaje significativo mantiene relación con conocimientos previos a diferencia del aprendizaje memorístico que solo consiste en la captación de información sin ningún tipo de conexión con otro conocimiento.

Las ventajas del aprendizaje significativo:

- Ayuda a la adquisición de conocimientos nuevos que mantienen relación con conocimientos previos de manera significativa, debido a que los alumnos son conscientes del conocimiento lo que les proporciona más claridad sobre un determinado tema.

- Los alumnos son conscientes del conocimiento, entienden mejor estructura cognitiva lo que les ayuda a interiorizar la nueva información.

- Al estar el nuevo conocimiento relacionado con el conocimiento anterior, el almacenamiento del conocimiento es más duradero (se aloja en la memoria a largo plazo) lo que se traduce en la retención de la información por más tiempo.

- Como el aprendizaje es personal, la percepción de los conocimientos será individual, así como los recursos utilizados para la creación del conocimiento.

- El aprender significativamente representa una manera sencilla mantener conocimientos.

Según Francisco (2008), existen diversos autores que explican la necesidad de promover en los estudiantes la toma de "decisiones en situaciones de incertidumbre" pero que esto no es lo que precisamente los docentes suelen enseñar. De acuerdo a Cohen citado por Francisco (2008), el estudiante de educación superior en los cuatro ámbitos fundamentales del proceso de socialización de acuerdo a la tecnología de la profesión, asimilar el rol y la cultura profesional así como relacionar este rol a los roles cotidianos. El estudiante durante su proceso de socialización debe aprender en cuatro esferas fundamentales: 1) la tecnología de la profesión, compuesta por lo que describe como los hechos (las habilidades y la teoría), 2) aprender a asimilar la cultura profesional, 3) encontrar una versión del rol que sea profesional y personalmente aceptable y 4) integrar ese rol profesional en todos los demás roles de la vida.

El poder empoderar el aprendizaje significativo en el aula origina en el estudiantado el $n$ cambio de actitud de forma esencial, debido a que se asume lo que aprenden, hay motivación, participan de manera activa, les gusta y se sienten participes del nuevo aprendizaje y con la experiencia educativa. Según (Ballester Vallori, 2005) “El cambio en el profesorado es inmediato, por lo que pasa de trabajar mucho y dedicar esfuerzo sin ver los resultados a trabajar en buena dirección evitándose el desgaste personal y muchísimo esfuerzo posterior con muy pocos resultados".

Para Ausubel (1976) el aprendizaje depende de la estructura cognitiva previa que se relaciona con la nueva información, cuando se habla de la estructura cognitiva, se está hablando de los conceptos, ideas que tienen los sujetos en un determinado campo del conocimiento, para poder orientar el aprendizaje es necesario saber cuál es la estructura cognoscitiva del estudiante, para definir cuáles son los conceptos y proposiciones que domina. Para (Ausubel J. N., 1976) “El aprendizaje significativo comprende la adquisición de nuevos significados y, a la inversa, éstos son producto del aprendizaje significativo. El surgimiento de nuevos significados en el alumno refleja la consumación de un proceso de aprendizaje significativo". 
Hay un aprendizaje significativo cuando los contenidos: Son relacionados de modo no arbitrario y sustancial no necesariamente deben ser aprendidos a pie de la letra o de forma memorística. El proceso educativo se debe relacionar lo que el individuo sabe para poder establecer la relación con lo que debe aprender. Para (Ausubel D., 1983), el aprendizaje significativo ocurre cuando una nueva información se conecta" con un concepto relevante ("subsunsor") pre existente en la estructura cognitiva, esto implica que, las nuevas ideas, conceptos y proposiciones pueden ser aprendidos significativamente en la medida en que otras ideas, conceptos o proposiciones relevantes estén adecuadamente claras y disponibles en la estructura cognitiva del individuo y que funcionen como un punto de "anclaje" a las primeras.

Según (Díaz Barriga Arceo \& Hernández Rojas, 2004) en todo caso, es evidente que el aprendizaje significativo es más importante y deseable que el repetitivo en lo que se refiere a situaciones académicas, ya que primero posibilita la adquisición de grandes cuerpos de conocimientos integrados, coherentes, estables, que tienen sentido para los alumnos, se puede decir entonces, que el aprendizaje significativo es el que orienta la creación de estructuras del conocimiento a través de la relación entre el conocimiento o ideas previas con la nueva información.

Pero no se puede considerar que el aprendizaje significativo solamente es la relación de la información nueva con la ya existe en la estructura cognoscitiva del que aprende, por el contrario, implica la transformación y avance de la nueva información, así como de la estructura cognoscitiva incluida en el aprendizaje.

En el Ecuador, la formación de los profesionales es llevada a cabo mediante un sistema gradual que instruye en virtud a funciones preventivas del sistema de educación superior "CES" (Consejo de Educación Superior). Las condiciones actuales en las que ha de desempeñarse el profesional, la globalización y la sociedad del conocimiento, plantean exigencias a la formación de profesionales universitarios, que permitan garantizar no sólo el dominio de los conocimientos para el desempeño específico de una profesión en un contexto histórico-social determinado, sino también y, fundamentalmente, la formación en valores, habilidades y recursos personales que motiven al egresado a desempeñarse con eficiencia, autonomía, ética y compromiso social en los contextos, heterogéneos y cambiantes, en donde va a desarrollarse- Todo ello exige a la universidad centrar la atención a la formación en competencias genéricas y profesionales.

Por su parte, los métodos utilizados en el mundo de las ciencias naturales por ejemplo para el aprendizaje son producto de instrumentos claves durante la formación "de personas competentes para el ejercicio de la profesión" (Escayola \& Vila, 2005 , p. 45). Al respecto señala que para que el diseño de una curricular sea idóneo es necesaria la participación de alumnos, profesionales recién egresados, docentes y la sociedad misma ya que permiten la interacción de los requerimientos colectivos del gremio a primera instancia por sus respectivos agentes, permitiendo predecir errores, mejorar el ámbito académico y docente.

Según (Rodríguez Torre, Avilez Miranda, Nicolalde Navarrete, Granda Encalada, \& Angulo Álvarez, 2017) El rol del docente debe ser, mediador del aprendizaje, que promueva una enseñanza auténtica donde las actividades instruccionales y de evaluación, sean secuenciadas y conectadas en el tiempo, planteando a los estudiantes problemas y conflictos a resolver cuyas condiciones contextuales (recursos y limitaciones, exigencia cognitiva, presión emocional, etc.), guarden una gran fidelidad con las condiciones contextuales que los estudiantes deberán afrontar en un futuro próximo. 
Conviene subrayar que este proceso de adaptación no es ajeno a la unción del docente como parte del proceso de formación, puesto que la constante actualización del docente facilitará el aprendizaje del alumno, ya que los conocimientos recibidos serán actuales lo que le evitará el desaprender conceptos desactualizados o ya obsoletos (Parra, 2016).

Un docente universitario, debe permitir un cambio y/o evolución en su rol de moderador del conocimiento (Escayola \& Vila, 2005), no solo en lo relacionado a la adquisición de nuevos conocimientos sino a nivel personal y colectivo, ya que de esta manera se logrará aumentar la motivación y el grado de responsabilidad del alumno. Cabe señalar que establecen que la aplicación de esta metodología a nivel grupal es considerablemente positiva en la formación de profesionales ya que responden al ejercicio propio de la profesión.

Se debe destacar que los estudiantes son capaces de aprender de manera autónoma cuando está motivado, cuando hay algo que le parece interesante, aprende observando, imitando escuchando y atendiendo las explicaciones, siguiendo instrucciones o trabajando de forma cooperativa, para expresa que (Romero Trenas, 2009)"el aprendizaje significativo a partir de dos elementos la actividad constructiva y la interacción con otros". La actividad constructiva se refiere a la actividad que realiza el estudiante y la interacción para la adquisición de los nuevos conocimientos.

Dentro de las disposiciones generales en el Marco Legal de la Educación Superior en el Ecuador, en la Constitución de la Republica, sobre la Educación Superior lo siguiente:

Artículo 350.- El sistema de educación superior tiene como finalidad la formación académica y profesional con visión científica y humanista; la investigación científica y tecnológica; la innovación, promoción, desarrollo y difusión de los saberes y las culturas; la construcción de soluciones para los problemas del país, en relación con los objetivos del régimen de desarrollo (Asamblea Nacional Constituyente del Ecuador, 2008, p. 162).

En el Reglamento de Régimen Académico se encuentran disposiciones que regulan entre otras la organización de los aprendizajes:

Artículo 11.- Organización del aprendizaje.La organización del aprendizaje consiste en la planificación del proceso formativo del estudiante, a través de actividades de aprendizaje con docencia, de aplicación práctica y de trabajo autónomo, que garantizan los resultados pedagógicos correspondientes a los distintos niveles de formación y sus modalidades (Consejo de Educación Superior, 2013, pág. 7).

Artículo 12.- Planificación y equivalencias de la organización del aprendizaje. - La organización del aprendizaje permiten la planificación curricular en un nivel de formación y en una modalidad específica de la educación superior.

Artículo 16.- Actividades de aprendizaje. La organización del aprendizaje se planificará incluyendo los siguientes componentes:

1. Componente de docencia. - Está definido por el desarrollo de ambientes de aprendizaje que incorporan actividades pedagógicas orientadas a la contextualización, organización, explicación y sistematización del conocimiento científico, técnico, profesional y humanístico.

\section{Estas actividades comprenderán:}

a. Actividades de aprendizaje asistido por el profesor. - Tienen como objetivo el desarrollo de conocimientos, habilidades, destrezas y valores, mediante clases presenciales $u$ otro ambiente de aprendizaje. Pueden ser conferencias, seminarios, orientación para estudio de casos, foros, clases en línea en tiempo sincrónico, docencia en servicio realizada en los escenarios 
laborales, entre otras. En las modalidades en línea y a distancia, el aprendizaje asistido por el profesor corresponde a la tutoría sincrónica.

b.- Actividades de aprendizaje colaborativo. - Comprenden el trabajo de grupos de estudiantes en interacción permanente con el profesor, incluyendo las tutorías. Están orientadas al desarrollo de la investigación para el aprendizaje y al despliegue de experiencias colectivas en proyectos referidos a temáticas específicas de la profesión. Son actividades de aprendizaje colaborativo, entre otras: la sistematización de prácticas de investigaciónintervención, proyectos de integración de saberes, construcción de modelos y prototipos, proyectos de problematización y resolución de problemas o casos. Estas actividades deberán incluir procesos colectivos de organización del aprendizaje con el uso de diversas tecnologías de la información y la comunicación, así como metodologías en red, tutorías in situ o en entornos virtuales.

2. Componente de prácticas de aplicación y experimentación de los aprendizajes. -Está orientado al desarrollo de experiencias de aplicación de los aprendizajes. Estas prácticas pueden ser, entre otras: actividades académicas desarrolladas en escenarios experimentales o en laboratorios, las prácticas de campo, trabajos de observación dirigida, resolución de problemas, talleres, manejo de base de datos y acervos bibliográficos. La planificación de estas actividades deberá garantizar el uso de conocimientos teóricos, metodológicos y técnico- instrumentales y podrá ejecutarse en diversos entornos de aprendizaje. Las actividades prácticas deben ser supervisadas y evaluadas por el profesor, el personal técnico docente y los ayudantes de cátedra y de investigación (Consejo de Educación Superior, 2013, pág. 8)
Basados en los componentes dispuestos por el Reglamento de Régimen Académico las actividades a realizar para la aplicación y experimentación de los aprendizajes que deben potenciar el contenido, método y mejorar las condiciones del proceso de aprendizaje, vinculando el fondo de experiencia con los nuevos saberes, por lo tanto es muy importante definir las metodologías y estrategias a fin de tener las herramientas pedagógicas, debido a que en la docencia superior se puede utilizar diversas formas de organización de la enseñanza en función de los objetivos que es planteados por el docente y de los recursos didácticos que se tiene a mano.

Cuando hablamos de método en el ámbito de la enseñanza nos referimos a la (Perdomo Rodríguez, 2016) "forma de proceder que tienen los profesores para desarrollar su actividad docente". Cada profesor concibe y ejecuta su tarea siguiendo fundamentalmente pautas basadas en sus ideas personales sobre la enseñanza o costumbres del gremio al que pertenece. A partir de las estrategias de aprendizaje. (p.5). Comprender y hacer uso de estrategias de aprendizaje permite y posibilita escenarios para la interacción, para intercambiar puntos de vistas hacia el conocimiento, para mantener una comunicación constante, para fortalecer la comunicación escrita y verbal, para potencializar el intercambio de ideas, dándoles la libertad a sus estudiantes de sentirse más cómodos en el momento de participar.

Los métodos de enseñanza en la Educación Superior, Lección Magistral, Estudio de casos, Resolución de problemas. Aprendizaje basado en problemas, Aprendizaje orientado a proyectos, Aprendizaje cooperativo, Contrato de aprendizaje. La educación Superior en el Contexto de la Universidades de Guayaquil y Agraria del Ecuador se aplican las siguientes modalidades de enseñanza como son: las clases teóricas, seminarios y talleres, clases prácticas, practicas externas, tutorías, 
trabajo en equipo, trabajo colaborativo, trabajo autónomo entre otros. Para (Ojeda \& Alcalá, 2004) Los profesores, al desarrollar las prácticas de enseñanza, conforman ciertos repertorios que les permiten interactuar con los estudiantes y configurar de cierto modo los ambientes destinados a la educación, esto posibilita identificar ciertas prácticas constantes en el desarrollo del proceso que van generado modalidades de enseñanza.

\section{CONCLUSIONES}

En el proceso de orientación del aprendizaje, es de vital importancia conocer la estructura cognitiva del alumno de la Educación Superior; no sólo se trata de saber la cantidad de información que posee, sino cuales son los conceptos y proposiciones que maneja, así como de su grado de estabilidad. Para Ausubel (1983), aprender es sinónimo de comprender e implica, como se ha dicho más arriba, una visión del aprendizaje basada en los procesos internos del alumno y no sólo en las respuestas externas. Con intención de promover la asimilación de saberes, en donde el docente de la Educación Superior utilice organizadores previos que favorezcan la creación de relaciones entre los saberes previos y los nuevos. Los organizadores tienen la finalidad de facilitar la enseñanza receptiva significativa, lo que permite que la exposición organizada de los contenidos propicie una mejor comprensión.

Para que el aprendizaje sea significativo se requiere de la disposición del estudiante de forma activa, se debe relacionar los conocimientos previos que se convierten en la piedra angular para la adquisición de los nuevos conocimientos, basados en una metodología coherente y la aplicación de estrategias que sean significativas tanto para el docente como para el estudiante, esta nueva información se debe incorporar a la estructura mental y genere o forme parte de la memoria comprensiva.

El análisis del aprendizaje significativo, ofreciendo el marco para el docente en el diseño de herramientas que permitan conocer la organización de la estructura cognitiva del educando, lo cual aprobará una orientación de la labor educativa en la Educación Superior. En consecuencia, emerge la importancia por saber cómo se adquieren los conocimientos para cuyo efecto tiene importancia central el proceso de desarrollo cognoscitivo y la estructura cognoscitiva del sujeto. No se puede imaginar a un Docente de la Educación Superior que trabaje con éxito y no sepa nada acerca del proceso del aprendizaje; en consecuencia el éxito se encuentra en disponer de la experiencia necesaria de los procesos cognoscitivos considerando que se está formando a un futuro profesionista y ciudadano al servicio de su país, por lo que el aprendizaje significativo es sumamente importante, añadiendo la educación dentro el concepto humanista y aplicada para el bien común, de manera que sus conocimientos adquiridos sean aplicados permanentemente en su vida y principalmente en su desempeño profesional destacándose como ciudadano útil al servicio del desarrollo del país.

\section{REFERENCIAS}

Asamblea Nacional Constituyente del Ecuador (2008). Constitución de la República del Ecuador (Versión de bolsillo). Quito, Ecuador: Asamblea Constituyente.

Ausubel, D. (1983). http://www.academia. edu/download/38902537/Aprendizaje_ significativo.pdf. Recuperado de http:// www.academia.edu/download/38902537/ Aprendizaje_significativo.pdf.

Ausubel, J. N. (1976). Psicología educativa. Un punto de vista cognoscitivo. Recuperado de http://cmapspublic2.ihmc.us/rid=1J3D72LMF1TF42P4PWD/aprendizaje\%20significativo.pdf. 
Ballester Vallori, A. (febrero de 2005). El Aprendizaje Significativo en la Práctica. Cómo hacer el aprendizaje significativo en el aula. SEDICl. Recuperado de http://sedici.unlp.edu. ar/handle/10915/24385

Baptista, M., \& et-al. (2014). Metodología de la investigación. México, México: McGraw-Hill / Interamericana Editores.

Baptista, M., Fernández, C., \& Hernández, R. (2014). Metodología de la investigación. México, México: McGraw-Hill / Interamericana Editores.

Canalejas, M., Cid, M., Martín, A., Martínez, M., Pineda, M., Soto, M., \& Vera, M. (2005). Estilos de aprendizaje en los estudiantes de enfermería. Educación Médica, 83-90.

Consejo de Educación Superior. (2013). Reglamento de régimen académico. Quito, Ecuador: Consejo de Educación Superior.

Díaz Barriga Arceo, F., \& Hernández Rojas, G. (2004). Estrategias docentes para un aprendizaje significativo. Una interpretación constructivista. México, México: McGraw-Hill.

Díaz, M. (2005). Modalidades de enseñanza centradas en el desarrollo de competencias. Oviedo: Ediciones Universidad de Oviedo.

Escayola, A., \& Vila, M. (2005). A las puertas del cambio en la formación universitaria. Educación Médica, 69-73.

Francisco, C. (2008). De la práctica de la enfermería a la teoría enfermera. Alcalá de Henares: Universidad de Alcalá

Gagné. (1965). Teoría del Aprendizaje
Ojeda, M., \& Alcalá, M. (2004). La enseñanza en las aulas universitarias. Una mirada desde las cátedras: aspectos curriculares que inciden en las prácticas pedagógicas de los equipos docentes. Revista Iberoamericana de Educación

Parra, J. (2016). Estrategias metodológicas de aprendizaje significativo en el módulo de enfermería del primer semestre "A" $\mathrm{y}$ "B" de la carrera de técnico superior en enfermería en el Instituto Tecnológico Superior Libertad período 2015-2016. Quito, Ecuador: Universidad Central del Ecuador

Perdomo Rodríguez, W. (marzo de 2016). Estudio de evidencias de aprendizaje significativo en un aula bajo el modelo flipped classroom. Edutec Revista Electrónica de Tecnología Educativa (55)

Rodríguez Torre, Ä., Avilés Miranda, J., Nicolalde Navarrete, L., Granda Encalada, V., \& Angulo Álvarez, M. (marzo de 2017). Cuando tenía todas las respuestas, me cambiaron las preguntas. Retos del profesorado universitario. Educación física y deportes, Revista Digital (226). Recuperado de http://www.efdeportes.com/ efd226/retos-del-profesorado-universitario. htm

Romero Trenas, F. (2009). El aprendizaje significativo y el constructivismo. Temas para la Educación Revista Digital para profesionales de la Enseñanza (3)

Zavalza (1991). Estrategias didácticas del aprendizaje 